\title{
EFECTOS A CORTO PLAZO DE LA CONTAMINACIÓN ATMOSFÉRICA SOBRE LA MORTALIDAD: RESULTADOS DEL PROYECTO EMECAM EN LA CIUDAD DE HUELVA, 1993-96 *
}

\author{
Antonio Daponte Codina (1), Pilar Gutiérrez-Cuadra (1), Ricardo Ocaña Riola (1), José Luis \\ Gurucelain Raposo (2), José Antonio Maldonado Pérez (3), Rosario Garrido de la Sierra (4), Javier \\ Serrano Aguilar (5), José María Mayoral Cortes (6). \\ (1) Escuela Andaluza de Salud Publica. Granada. \\ (2) Delegación Provincial de Salud, Consejería de Salud. Huelva. \\ (3) Hospital General de Huelva, Servicio Andaluz de Salud. Huelva. \\ (4) Distrito Sanitario de Huelva, Servicio Andaluz de Salud. Huelva. \\ (5) Consejería de Medio Ambiente. Sevilla. \\ (6) Distrito Sanitario de Camas, Servicio Andaluz de Salud. Sevilla. \\ $\left(^{*}\right)$ Este trabajo cuenta con una beca del Fondo de Investigaciones Sanitarias (Expediente núm 97/0051-07) y por \\ la Consejería de Salud de la Junta de Andalucía.
}

\section{RESUMEN}

Fundamento: El objetivo del estudio fue estimar la asociación entre los niveles de contaminación atmosférica y la mortalidad diaria en la ciudad de Huelva para el período 1993-1996 utilizando la metodologia EMECAM.

Métodos: Se utilizaron como indicadores de mortalidad el número de muertos diarios por todas las causas menos externas, la mortalidad en sujetos mayores de 69 años, la mortalidad por enfermedades del aparato circulatorio y la mortalidad por enfermedades respiratorias. Se analizaron cuatro contaminantes, $\mathrm{SO}_{2}, \mathrm{PM}_{10}, \mathrm{NO}_{2}, \mathrm{y} \mathrm{CO}$, cuyos niveles diarios se obtuvieron de la red de vigilancia de la contaminación atmosférica en Huelva. Se construyeron modelos de regresión autoregresiva de Poisson controlando por tendencia, estacionalidad, temperatura, humedad, gripe, y eventos inusuales.

Resultados: Para la mortalidad por todas las causas se halló un efecto significativo del $\mathrm{NO}_{2}$ para todo el período (RR $10 \mu \mathrm{gr} / \mathrm{m}^{3}: 1,0414$; IC95\%: 1,0047-1,0794) y de las partículas $\left(\mathrm{PM}_{10}\right)$ para el semestre frío $\left(\mathrm{RR}_{10} \mu \mathrm{gr} / \mathrm{m}^{3}: 1,0358\right.$; $\mathrm{IC} 95 \%$ : 1,0007-1,0722). Para la mortalidad en mayores de 69 años se obtuvo una asociación significativa con el $\mathrm{SO}_{2}$ para todo el período $\left(\mathrm{RR}_{10} \mu \mathrm{gr} / \mathrm{m}^{3}\right.$ : 1,0606; IC95\%: 1,0020-1,1227). Sc halló una asociación significativa para la mortalidad por enfermedades respiratorias con las partículas $\left(\mathrm{PM}_{10}\right)$ en el semestre frio $\left(R_{10} \mu \mathrm{gr} / \mathrm{m}^{3}: 1,1412\right.$; IC95\%: 1,0300-1,2644). No se obtuvieron asociaciones significativas para la mortalidad por enfermedades del aparato circulatorio con ningún contaminantes, ni tampoco se observó ningún efecto significativo de los niveles de $\mathrm{CO}$ en los indicadores de mortalidad.

Correspondencia:

Antonio Daponte Codina.

Escuela Andaluza de Salud Pública.

Campus Universitario de Cartuja.

Apdo Correos 2070.

18080 Granada.

Tclf: 958161044

Fax: 958161142

Correo electrónico: daponte@easp.es

\section{ABSTRACT \\ The Short-term Impact of Air Pollution on the Mortality. Results of the $E M E C A M$ Project in the city of Huelva, 1993-1996}

Background: The objective of this study was to estimate the relationship between the levels of air pollution and the daily mortality in the city of Huelva for the 1993-1996 period using the EMECAM methodology.

Methods: The number of daily deaths for all causes except external ones, the death rate of those over age 69 , due to diseases of the circulatory system and for respiratory diseases were used as rate indicators. Four pollutants $-\mathrm{SO}_{2}$, $\mathrm{PM}_{10}, \mathrm{NO}_{2}$ and $\mathrm{CO}$ - were analyzed, the daily levels of which were furnished by the air pollution monitoring network in Huelva. Autoregressive Poisson regression models were constructed controlling by tendency, seasonality, temperature, humidity, flue and events out of the ordinary.

Results: For the mortality rate for all causes, a significant association impact was found to exist for the $\mathrm{NO}_{2}$ for the entire period $\left(\mathrm{RR}_{10} \mu \mathrm{g} / \mathrm{m}^{3}: 1.0414 ; \mathrm{CI} 95 \%: 1.0047-1.0794\right)$ and for the particles $\left(\mathrm{PM}_{10}\right)$ for the cold half of the year $\left(R_{10} \mu \mathrm{g} / \mathrm{m}^{3}: 1.0358\right.$; CI95\%: 1.007-1.0722). For the mortality in people over age 69, a significant relationship was found to exist for $\mathrm{SO}_{2}$ throughout the entire period $\left(R_{10} \mu \mathrm{g} / \mathrm{m}^{3}\right.$ : 1.0606 ; C195\%: 1.0020-1.1227). A significant relationship to the mortality from respiratory disease particles $\left(\mathrm{PM}_{10}\right)$ was found to exist for the cold half of the year (RR $10 \mu \mathrm{g} / \mathrm{m}^{3}$ : 1.1412 ; IC95\%: 1.0300-1.2644). There was no association of contaminants with cardiovascular mortality; also there was no association between levels of $\mathrm{CO}$ and mortality indicators. 
Conclusiones: En Huelva se han encontrado asociaciones significativas entre los niveles actuales de contaminación atmostérica por partículas, $\mathrm{SO}_{2}, y \mathrm{NO}_{2}$, y la mortalidad diaria. $\mathrm{E}$ efecto de estos contaminantes sobre la mortalidad es coherente con la literatura científica, aunque en el caso de Huelva, el escaso número de defunciones diarias debido a su pequeña población y otros factores limitan su consistencia.

Palabras clave: Contaminación atmosférica. Mortalidad. Series temporales. Estudio multicéntrico.
Conclusions: In Huelva, significant relationships have been found to exist between the current levels of air pollution resulting from particles, $\mathrm{SO}_{2}$ and $\mathrm{NO}_{2}$ and the daily mortality. The impact of these pollutants on the mortality is coherent with scientific literature, although in the case of Huelva, the extremely small number of daily deaths due to its small population and other factors limit the consistency thereof.

Key words: Air pollution. Mortality. Time series. Mutticenter study.

\section{INTRODUCCIÓN}

Huelva es una ciudad industrial cuyo desarrollo se produce a partir de la segunda mitad del siglo XIX, ligado a la fuerte actividad minera en la provincia y a su puerto de $\operatorname{mar}^{1}$. Desde principios de la década de los años 60 , Huelva cuenta con una potente industria química que ha generado en el pasado un importante impacto ambiental sobre el medio natural de la ciudad. Durante la década de los 70 , cuando se puso en funcionamiento la red de vigilancia de la contaminación atmosférica y hubo por lo tanto información oficial disponible, fueron frecuentes las situaciones de emergencia por superación de los niveles normativos de inmisión. En los primeros años ochenta los niveles «anuales» medios de inmisión para $\mathrm{SO}_{2}$ llegaron a superar, en alguna estación captadora del centro urbano, los 100 $\mu \mathrm{gr} / \mathrm{m}^{3}$, siendo frecuentes las situaciones de alerta $^{2.3}$. La proximidad del polo químico al centro urbano, los elevados niveles de contaminación atmosférica, junto con la existencia de frecuentes situaciones de alerta, han determinado en el pasado que la contaminación atmosférica fuera el problema más preocupante de la ciudad para sus habitantes. ${ }^{4}$. A principios de la década de los 80 se cerró la central térmica responsable de más del $30 \%$ de las emisiones de $\mathrm{SO}_{3}$, y se introdujeron medidas urgentes para disminuir los niveles de contaminación, que se continuaron en 1990 con la puesta en marcha del Plan Corrector de la Contaminación Atmosférica de Huelva, permitiendo todo ello la disminución progresiva de los niveles de inmisión de los principales contaminantes de origen industrial ${ }^{5}$.

A pesar de la evidente relevancia de la contaminación atmosférica para la salud pú- blica en Huelva, la evaluación científica del impacto de los niveles de inmisión en la morbilidad o mortalidad ha sido escasa. Así, se ha estudiado el impacto de la contaminación atmosférica sobre especies del medio natural, como forma indirecta de conocer la dinámica de los contaminantes a lo largo del tiempo y estimar los niveles de exposición de la población ${ }^{6}$. Además, se ha valorado la posible asociación de los niveles de contaminación atmosférica con la morbilidad respiratoria ${ }^{7}$. En 1990 se realizó un estudio del impacto de los niveles de contaminantes en la mortalidad; en dicho estudio se estimaron las correlaciones entre mortalidad y uso de servicios de urgencias, y niveles diarios de inmisión de partículas (humos), óxidos de nitrógeno y $\mathrm{SO}_{2}{ }^{8}$. Los resultados del mismo revelaron una correlación positiva entre la mortalidad y uso de urgencias, y los niveles de NOx y $\mathrm{NO}_{2}$, pero no así con humos o el $\mathrm{SO}_{2}$, aun cuando las limitaciones en la serie temporal, la calidad de los datos y el abordaje metodológico limitan sus conclusiones.

El objetivo de este estudio es evaluar los efectos agudos de la contaminación atmosférica sobre la mortalidad en la ciudad de Huelva, utilizando la metodología EMECAM.

\section{MATERIAL Y METODOS}

La ciudad de Huelva tiene una población de 144.579 personas según el censo de 1991. El período de estudio fue 1993-1996, por ser para dichos años para los que existían datos de mortalidad y datos de los niveles de los contaminantes con las características de calidad establecidas en el protocolo EMECAM ${ }^{9}$. El número de defunciones diarias ocurridas 
entre los residentes de Huelva fueron tomados del Registro de Mortalidad de Andalucía, excluyéndose las muertes que ocurrieron fuera de la ciudad. Los niveles diarios de los contaminantes proceden de la Red de vigilancia de la contaminación atmosférica facilitados por la Consejería de Medio Ambiente. En 1996 la Red de Inmisión del Aire de la Agencia de Medio Ambiente estaba compuesta por 6 estaciones captadoras automáticas. De estas, cinco estaciones disponían de series temporales de al menos tres años, y de ellas, en tres estaciones para las partículas $\mathrm{PM}_{10}$ y en cuatro para el $\mathrm{SO}_{2}, \mathrm{CO}$, y $\mathrm{NO}_{2}$ el número de datos válidos fueron superiores al $75 \%$. Las técnicas analíticas de los sensores automáticos de las estaciones captadoras finalmente seleccionadas, Estadio, Rosales, Marismas del Titán, y Pozo Dulce, fueron la atenuación de la radiación beta para las partículas $\left(\mathrm{PM}_{10}\right)$, la absorción infrarroja para el $\mathrm{CO}$, la quimiluminiscencia para el $\mathrm{NO}_{2}$, y la fluorescencia ultravioleta para el $\mathrm{SO}_{2}$. La temperatura media diaria $\left({ }^{\circ} \mathrm{C}\right)$ y la humedad relativa $(\%)$ se estimaron a partir de los datos del Instituto Nacional de Meteorología. El número de casos semanales de gripe se obtuvo del sistema de Enfermedades de Declaración Obligatoria de Andalucía, y fueron facilitados por las Delegaciones Provinciales de
Salud, realizándose una estimación posterior de casos diarios.

El análisis cstadístico de datos se realizó siguiendo la metodología $E M E C A M^{9,10}$ evaluándose el efecto de cada contaminante en todo el período, en el período frío (desde noviembre hasta abril) y en el período cálido (desde mayo hasta octubre), para cada indicador de mortalidad, mediante modelos de regresión de Poisson. Posteriormente, se estimó el Riesgo Relativo (RR) y su intervalo de confianza al $95 \%$ para la mortalidad por todas las causas menos las externas, mortalidad por todas las causas menos las externas en mayores de 69 años, mortalidad por enfermedades respiratorias y mortalidad por enfermedades del aparato circulatorio, correspondientes a un incremento de 10 unidades en el contaminante.

\section{RESULTADOS}

El número medio de muertes diarias por todas las causas menos las externas en Huelva ha sido de 2,59, de 1,77 en mayores de 69 años, de 1,01 por enfermedades del aparato circulatorio, y de 0,3 por enfermedades respiratorias, y en todas las causas estudiadas hubo días sin mortalidad (tabla 1). Para la

Tabla 1

Descripción de las causas de mortalidad, Huelva 1993-1996

\begin{tabular}{|lllccccccc|}
\hline Causa de Mortalidad & Periodo & Minimo & Máximo & Media & $\begin{array}{c}\text { Desviación } \\
\text { estándar }\end{array}$ & Percentil 10 & Mediana & Percentil 90 \\
\hline \multirow{2}{*}{ Todas las causas } & Todo & 0 & 9 & 2,59 & 1,65 & 1 & 2 & 5 \\
& Cálido & 0 & 9 & 2,34 & 1,59 & 1 & 2 & 4 \\
& Frío & 0 & 9 & 2,84 & 1,68 & 1 & 3 & 5 \\
\hline \multirow{2}{*}{ Mayores de 70 años } & Todo & 0 & 8 & 1,77 & 1,36 & 0 & 2 & 4 \\
& Cálido & 0 & 8 & 1,58 & 1,26 & 0 & 1 & 3 \\
& Frío & 0 & 8 & 1,97 & 1,44 & 0 & 2 & 4 \\
\hline \multirow{2}{*}{ Aparato Circulatorio } & Todo & 0 & 6 & 1,01 & 1,02 & 0 & 1 & 2 \\
& Cálido & 0 & 5 & 0,86 & 0,91 & 0 & 1 & 2 \\
& Frío & 0 & 6 & 1,15 & 1,11 & 0 & 1 & 3 \\
\hline \multirow{2}{*}{ Respiratorias } & Todo & 0 & 3 & 0,30 & 0,54 & 0 & 0 & 1 \\
& Cálido & 0 & 3 & 0,28 & 0,52 & 0 & 0 & 1 \\
\hline
\end{tabular}


mortalidad por todas las causas el número de muertos diarios fue de al menos 1 en el $90 \%$ de los días del período de estudio, con un máximo de 9 . Sin embargo, para la mortalidad por enfermedades respiratorias tan sólo en un $10 \%$ de los días del período hubo al menos 1 muerto, estando el máximo en tres para estos días. Por estaciones del año, la media de muertes diarias fue en general mayor en el semestre frío que en el cálido para todas las causas estudiadas, siendo la diferencia en la media de muertes diarias para todas las causas menos externas de un $21 \%$ más en el semestre frío, de un $25 \%$ más para los mayores de 70 , de un $34 \%$ más por enfermedades del aparato circulatorio, y de un $11 \%$ más por enfermedades respiratorias.

Las tendencias de los contaminantes para todo el período se recogen en la figura 1 . Los niveles de los contaminantes en Huelva, recogidos en la tabla 2, no superan los valores de calidad del aire establecidos por la OMS ${ }^{11}$. Destaca también el hecho de que no existen diferencias apreciables en las medias diarias entre el semestre cálido y el frío, salvando el $\mathrm{CO}$, para el que los niveles son casi un $50 \%$ mayores en el semestre frío. Para los otros contaminantes los niveles son incluso ligeramente superiores en el semestres cálido que en el frío. Las correlaciones entre las estaciones captadoras para cada contaminante fueron positivas y significativas variando entre 0,20 y 0,60 para el $\mathrm{SO}_{2}, 0,06$ y 0,29 para $\mathrm{PM}_{10}$, 0,12 y 0,44 para el $\mathrm{NO}_{2}$, y 0,08 y 0,35 para el CO. Las correlaciones entre contaminantes se recogen en la tabla 3, variando en un rango de 0,075 a 0,310. Las correlaciones más altas entre estaciones captadoras se han obtenido para el $\mathrm{SO}_{2}$, lo cuál, pucde cstar justificado, además de por fenómenos locales en la dinámica de los contaminantes, por la ubicación de los sensores. La red de vigilancia de la contaminación en Huelva se diseñó inicialmente para detectar aumentos rápidos de los niveles de inmisión, particularmente para detectar situaciones de alerta, debidos a emisiones, sobre todo de $\mathrm{SO}_{2}$, provenientes del polo químico vecino al núcleo urbano. Es por lo que todavía hoy, la mayoría de los sensores, y particularmente los que se pudieron utilizar en este estudio, están distribuidos en forma de pantalla (figura 2) en la zona urbana colindante con la zona industrial y no cubren la totalidad del área que ocupa el núcleo urbano. Esto implica además que la distribución de sensores sería inadecuada para algunos contaminantes de origen no industrial, particularmente los generados por el tráfico de vehículos en el núcleo urbano. Por lo tanto, la distribución de los sensores podría afectar a la capacidad del estudio para identificar asociaciones entre los niveles de contaminación y la mortalidad en Huelva.

La tabla 4 recoge la asociación entre los niveles de los contaminantes y la mortalidad, expresada como el riesgo relativo asociado a incrementos de $10 \mu \mathrm{gr} / \mathrm{m}^{3}$ del contaminante, exceptuando el $\mathrm{CO}$ que se expresa en aumentos de $10 \mathrm{mgr} / \mathrm{m}^{3}$. El $\mathrm{SO}_{2}$ está asociado de forma positiva y significativamente con la mortalidad en mayores de 69 años y cercano a la significación con todas las causas menos externas $(p<0,07)$, siendo el retardo del mismo día el de mejor ajuste en ambos casos. Las partículas están asociadas positivamente con todos los indicadores de mortalidad en todo el período, pero no de forma significativa; la asociación resulta sin embargo significativa con la mortalidad por todas las causas menos externas en el semestre frío $\left(\mathrm{RR}_{10} \mu \mathrm{gr} / \mathrm{m}^{3}\right.$ : 1,0358; IC95\%: 1,0007-1,0722) y en el retardo 5 , y con las enfermedades respiratorias también en el semestre frío $\left(\mathrm{RR}_{10}\right.$ $\mu \mathrm{gr} / \mathrm{m}^{3}$ : 1,1412; IC95\%: 1,0300-1,2644) y en el retardo $1 . \mathrm{E}_{1} \mathrm{NO}_{2}$ está asociado positiva $y$ significativamente con la mortalidad por todas las causas menos externas para todo el período en el retardo 5 , y el $\mathrm{CO}$ está asociado positiva aunque no significativamente con todos los indicadores menos la mortalidad por enfermedades respiratorias. 
Figura 1

Tendencias en los niveles diarios de contaminación atmosférica, Huelva 1993-1996

Dióxido de azufre

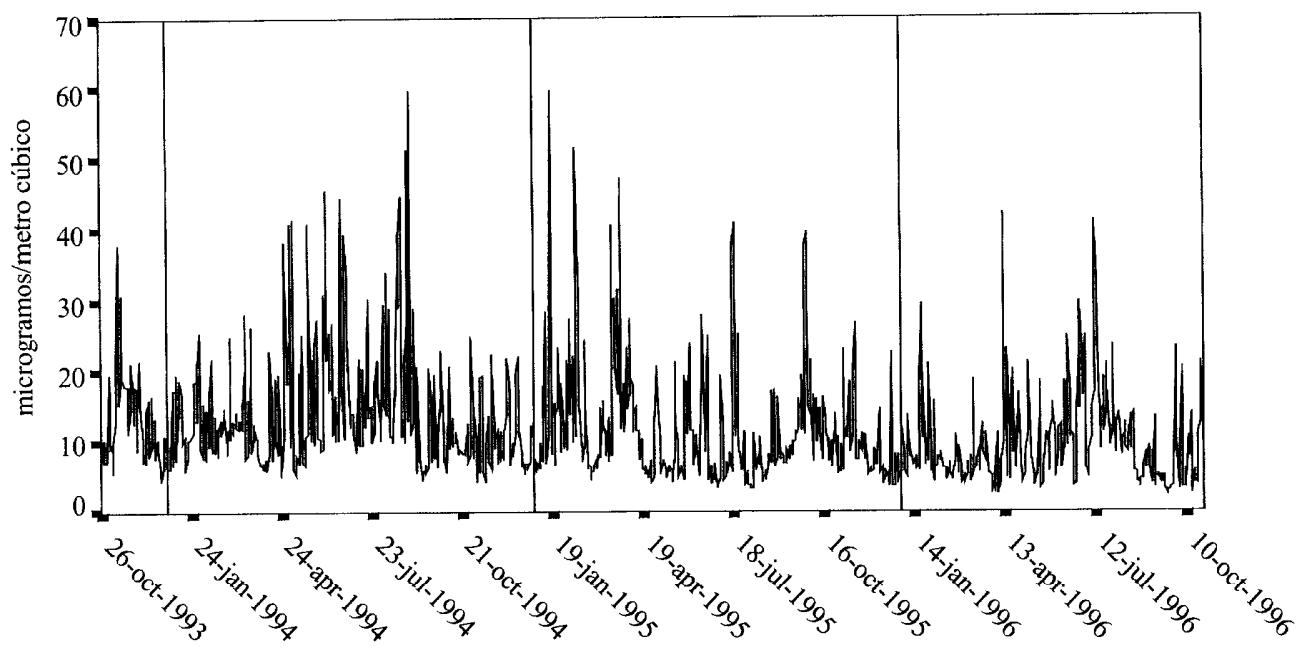

Tiempo

Dióxido de nitrógeno

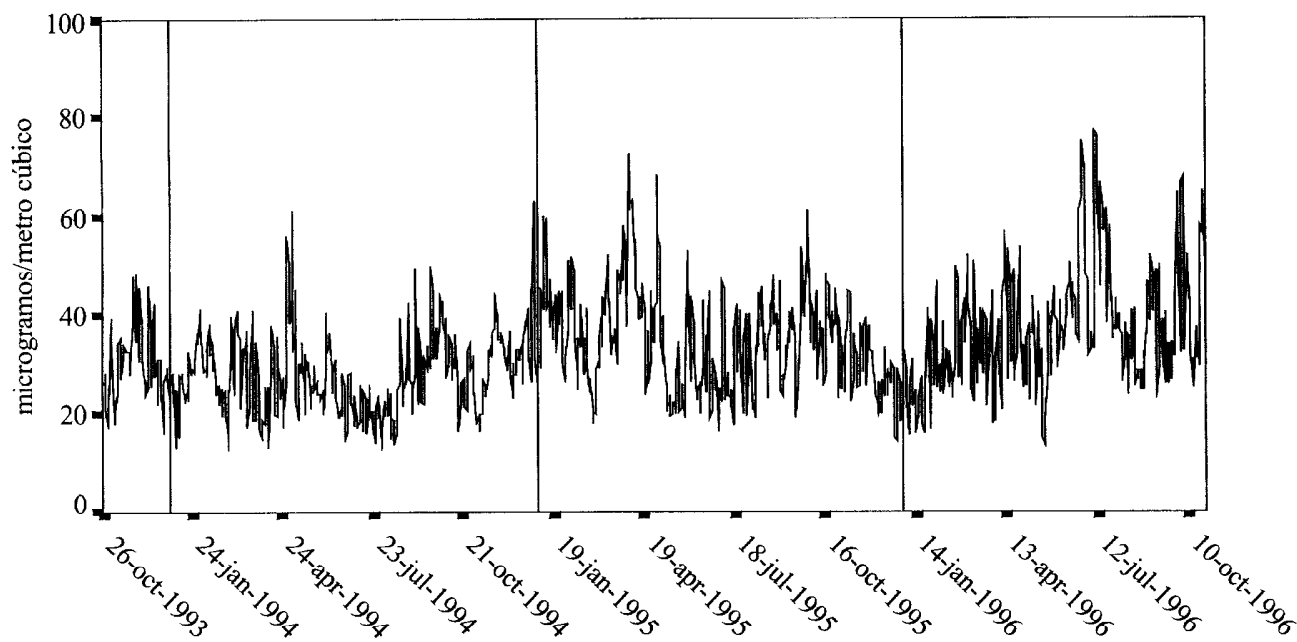

Tiempo 
Figura 1 (continuación)

Tendencias en los niveles diarios de contaminación atmosférica, Huelva 1993-1996

Partículas

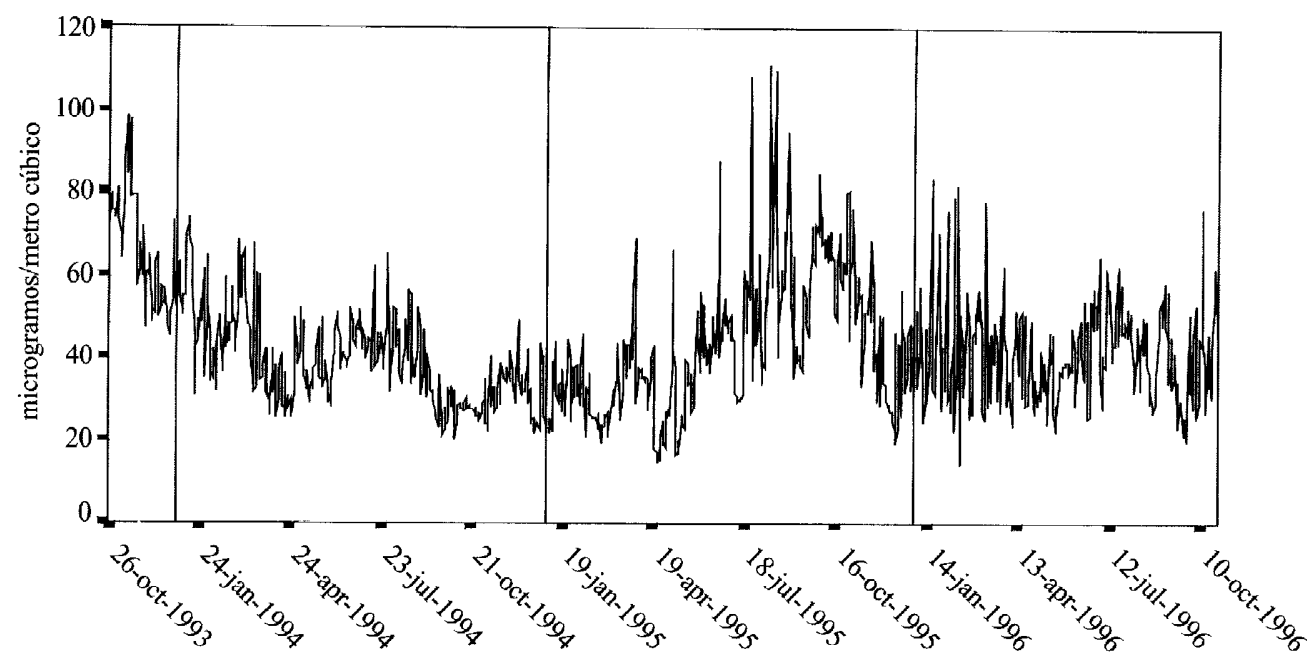

Tiempo

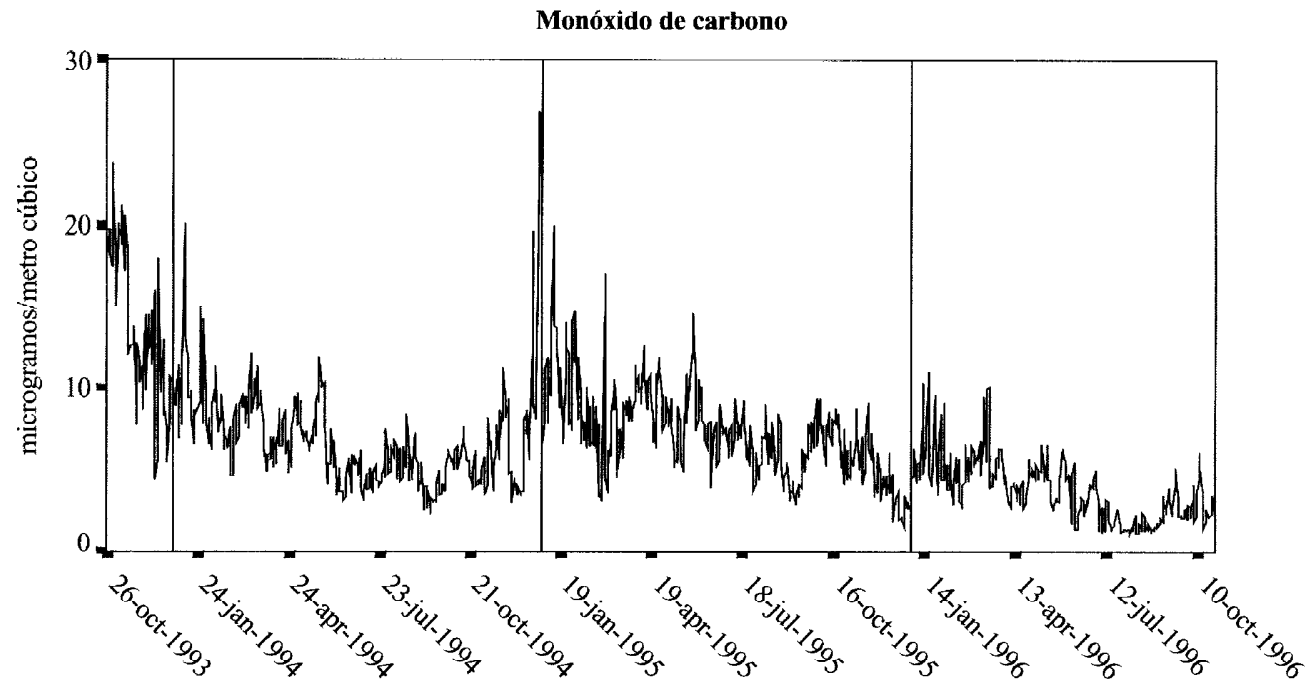

Tiempo 
Tabla 2

Descripción de los niveles de temperatura y contaminantes para 24 horas, Huelva 1993-1996

\begin{tabular}{|c|c|c|c|c|c|c|c|c|}
\hline Variables & Periodo & Minimo & Máximo & Media & $\begin{array}{l}\text { Desviación } \\
\text { estándar }\end{array}$ & Percentil 10 & Mediana & Percentil 90 \\
\hline \multirow[t]{3}{*}{ Temperatura media $\left({ }^{\circ} \mathrm{C}\right)$} & Todo & 5,50 & 32,5 & 18,35 & 5,51 & 11,29 & 18,20 & 25,80 \\
\hline & Cálido & 13.00 & 32,50 & 22,69 & 3,48 & 18,24 & 22,40 & 27,00 \\
\hline & Frio & 5,50 & 22,50 & 13,93 & 3,20 & 9,95 & 13,90 & 18,10 \\
\hline \multirow[t]{3}{*}{$\mathrm{SO}_{2}\left(\mu \mathrm{gr} / \mathrm{m}^{3}\right)$} & Todo & 2,3 & 59,7 & 11,7 & 7,6 & 4,9 & 9,8 & 21,1 \\
\hline & Cálido & 2,5 & 59,7 & 12,0 & 8,3 & 4,4 & 10,0 & 22,6 \\
\hline & Frío & 2,3 & 59,6 & 11,3 & 6,9 & 5,2 & 9,4 & 19,6 \\
\hline \multirow[t]{3}{*}{$\mathrm{PM}_{10}\left(\mu \mathrm{gr} / \mathrm{m}^{3}\right)$} & Todo & 14,1 & 111,1 & 42,5 & 15 & 26,1 & 40,0 & 63,3 \\
\hline & Cálido & 16,3 & 111,1 & 43,4 & 14,7 & 27,3 & 41,4 & 63,6 \\
\hline & Frio & 14,1 & 98,9 & 41,6 & 15,2 & 25,0 & 38,4 & 62,9 \\
\hline \multirow[t]{3}{*}{$\mathrm{NO}_{2}\left(\mu \mathrm{gr} / \mathrm{m}^{3}\right)$} & Todo & 12,3 & 77,0 & 32,9 & 10,9 & 20,1 & 31,5 & 46,6 \\
\hline & Cálido & 12,3 & 77,0 & 33,4 & 11,7 & 19,9 & 32,0 & 48,3 \\
\hline & Frío & 12,3 & 72,5 & 32,3 & 9,9 & 20,2 & 31,0 & 45,4 \\
\hline \multirow[t]{3}{*}{$\mathrm{CO}\left(10 \mathrm{mgr} / \mathrm{m}^{3}\right)$} & Todo & 1,2 & 26,9 & 6,6 & 3,7 & 2,7 & 6,1 & 10,6 \\
\hline & Cálido & 1,2 & 19,7 & 5,3 & 2,7 & 1,9 & 5,1 & 8,1 \\
\hline & Frío & 1,5 & 26,9 & 7,9 & 4,0 & 3,8 & 7,4 & 12,7 \\
\hline
\end{tabular}

Tabla 3

Coeficientes de correlación de Pearson para niveles de contaminantes de 24 horas, Huelva 1993-1996

\begin{tabular}{|c|c|c|c|c|}
\hline & $\mathrm{SO}_{2}\left(\mu \mathrm{gr} / \mathrm{m}^{3}\right)$ & $P M_{10}\left(\mu \mathrm{gr} / \mathrm{m}^{3}\right)$ & $\mathrm{NO}_{2}\left(\mu \mathrm{gr} / \mathrm{m}^{3}\right)$ & $\mathrm{CO}\left(\mathrm{mgr} / \mathrm{m}^{3}\right)$ \\
\hline $\mathrm{SO}_{2}\left(\mu \mathrm{gr} / \mathrm{m}^{3}\right)$ & 1,000 & $0,241^{* *}$ & $0,310^{* *}$ & $0,157^{* *}$ \\
\hline $\mathrm{PM}_{10}\left(\mu \mathrm{gr} / \mathrm{m}^{3}\right)$ & $0,241^{* *}$ & 1,000 & $0,229^{* *}$ & $0,308 * *$ \\
\hline $\mathrm{NO}_{2}\left(\mu \mathrm{gr} / \mathrm{m}^{3}\right)$ & $0,310^{* *}$ & $0,229 * *$ & 1,000 & $0,075 *$ \\
\hline $\mathrm{CO}\left(\mathrm{mgr} / \mathrm{m}^{3}\right)$ & $0,157^{* *}$ & $0,308^{* *}$ & $0,075^{*}$ & 1,000 \\
\hline
\end{tabular}

**. Valor pp<0,01*, Valor pp<0,05.

Los incrementos en la mortalidad debidos al efecto de los niveles de contaminantes resultan muy moderados: para incrementos de $10 \mu \mathrm{gr} / \mathrm{m}^{3}$ del contaminante se produciría un aumento de la mortalidad por todas las causas del orden del $4 \%$ debido al $\mathrm{NO}_{2}$, y del $3,5 \%$ en el semestre frío debido a las partículas; el $\mathrm{SO}_{2}$ incrementaría la mortalidad en mayores de 69 años en un 6\%; y las partículas producirían un incremento de la mortalidad por enfermedades respiratorias en el semestre frío de un 14\%. De la valoración de los retardos de mejor ajuste en cada caso, se desprende que el efecto sobre la mortalidad de los niveles de partículas y $\mathrm{SO}_{2}$ para la mortalidad por todas las causas y para mayores de 69 años se produce en el mismo día, siendo sin embargo debido a la contaminación del $2 .^{\circ}$ al $5 .^{\circ}$ día anteriores para las causas específicas. En el caso del $\mathrm{NO}_{2}$ y del $\mathrm{CO}$ el efecto principal tiende a producirse con los niveles de contaminación de días anteriores, particularmente para el $\mathrm{CO}$, cuyo mejor ajuste se produce para aumentos de los niveles de contaminación del $3 .^{\circ}$ y $5 .^{\circ}$ días anteriores. 
Figura 2

Distribución de los sensores de contaminación atmosférica en la ciudad de Huelva

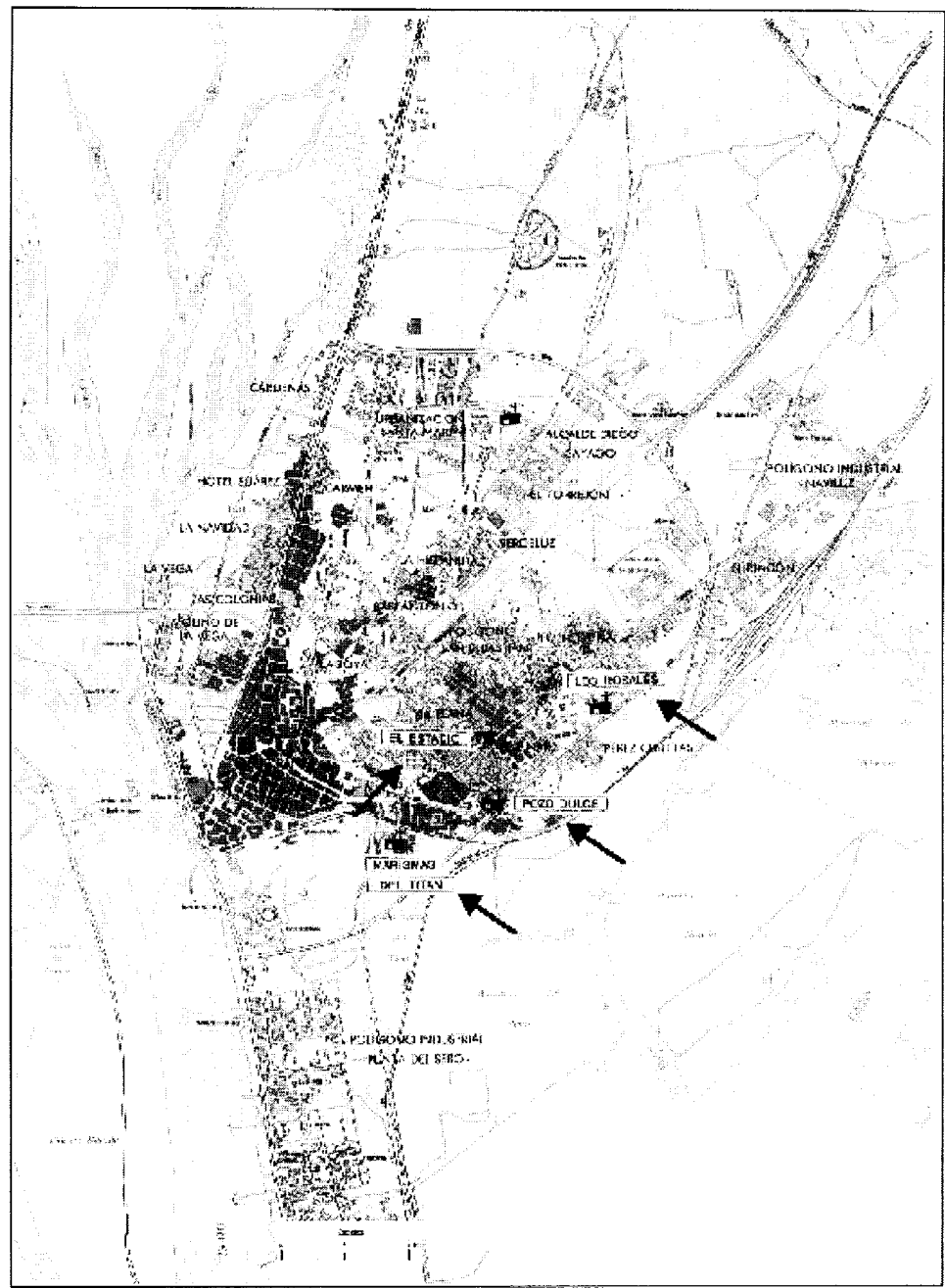

Tabla 4

Asociación entre contaminación atmosférica y mortalidad en Huelva, 1993-1996

\begin{tabular}{|c|c|c|c|c|c|c|c|c|}
\hline & \multicolumn{2}{|c|}{$\begin{array}{l}\text { Mortalidad por todas } \\
\text { las causas }\end{array}$} & \multicolumn{2}{|c|}{ Mortalidad mayores de 69 años } & \multicolumn{2}{|r|}{$\begin{array}{c}\text { Mortalidad } \\
\text { Aparato Circulatorio }\end{array}$} & \multicolumn{2}{|c|}{$\begin{array}{c}\text { Mortalidad } \\
\text { Enfermedades Respiratorias }\end{array}$} \\
\hline & $R$ & $R R_{\prime n} \mu g r / m^{3}(I C 95 \%)$ & $R$ & $R R_{/} \mu g r / m^{3}(I C 95 \%)$ & $R$ & $R R_{I 0 H} \mathrm{Hgr} / \mathrm{m}^{3}(\mathrm{IC} 95 \%)$ & $R$ & $R R_{10} \mu g r / m^{3}$ (IC 95\%) \\
\hline $\mathrm{SO}_{2}\left(\mu \mathrm{gr} / \mathrm{m}^{3}\right)$ & 0 & $1,0356(0,9872-1,0863)$ & 0 & $1,0606(1,0020-1,1227)$ & 3 & $0,9430(0,8678-1,0247)$ & 2 & $0,9065(0,7782-1,0561)$ \\
\hline $\mathrm{PM}_{10}\left(\mu \mathrm{gr} / \mathrm{m}^{3}\right)$ & 0 & $1,0249(0,9979-1,0526)$ & 0 & $1,0282(0,9951-1,0624)$ & 5 & $1,0305(0,9885-1,0742)$ & 3 & $1,0765(0,9936-1,1664)$ \\
\hline $\mathrm{NO}_{2}\left(\mu \mathrm{gt} / \mathrm{m}^{3}\right)$ & 5 & $1,0414(1,0047-1,0794)$ & 2 & $0,9781(0,9354-1,0228)$ & 3 & $0,9658(0,9094-1,0257)$ & 2 & $0,9187(0,8245-1,0236)$ \\
\hline $\mathrm{CO}\left(\mathrm{mg} / \mathrm{m}^{3}\right)^{*}$ & 3 & $1,0044(0,9923-1,0166)$ & 5 & $1,0075(0,9932-1,0220)$ & 5 & $1,0143(0,9962-1,0327)$ & 5 & $0,9855(0,9491-1,0233)$ \\
\hline
\end{tabular}

R: Retardo.

$\mathrm{RR}_{10} \mu \mathrm{gr} / \mathrm{m}^{3}$ (IC 95\%): Riesgo relativo de mortalidad e intervalo de confianza del $95 \%$ para incrementos de $10 \mu \mathrm{gr} / \mathrm{m}^{3}$ del contaminante.

(*): Para el CO los incrementos son de $10 \mathrm{mg} / \mathrm{m}^{3}$. 
La falta rclativa de consistencia en los retardos de mejor ajuste, y en el sentido y magnitud de las asociaciones de cada contaminante con los diferentes indicadores de mortalidad, puede estar condicionado por el escaso número de muertes diarias que se producen en Huelva debido a su pequeño tamaño. Así, en términos generales, las partículas y el $\mathrm{SO}_{2}$ resultan en asociaciones positivas e incluso significativas con los dos indicadores de mortalidad general, todas las causas y mayores de 69 años, y pierden la significación y algunas son negativas para las causas específicas. El efecto del $\mathrm{NO}_{2}$ resulta parecido al de los otros dos contaminantes, teniendo una asociación positiva y significativa con la mortalidad por todas las causas y perdiendo la significación y haciéndose negativa con causas más específicas. Otro fenómeno que podría influir en esta inestabilidad de las asociaciones es la temperatura; al igual que ocurre en otras ciudades del sur ${ }^{12}$, el posible impacto de la temperatura sobre la mortalidad, debido en forma de ola de calor a la llegada del verano y a los elevados valores medios que se alcanzan, así como su interacción con los contaminantes, dificulta la identificación de la relación entre contaminantes y mortalidad ajustando por el efecto de la temperatura, cuando como en este estudio se utilizan métodos paramétricos.

\section{CONCLUSIONES}

Como conclusión, en Huelva se han encontrado asociaciones positivas y en algunos casos significativas entre los niveles de contaminación atmosférica y la mortalidad, particularmente del $\mathrm{SO}_{2}, \mathrm{NO}_{2}$ y partículas, con los indicadores generales de mortalidad principalmente, la mortalidad por todas las causas y en mayores de 69 años, y también en el semestre frío con la mortalidad por enfermedades respiratorias. Al menos tres fenómenos condicionan la interpretación de estos resultados: la propia distribución de los sensores disponibles que dificultaría la identificación de asociaciones entre los con- taminantes y la mortalidad, el escaso número de muertos diarios, particularmente por causas específicas, que disminuye el poder del estudio para encontrar asociaciones, y el posible impacto de la temperatura sobre la mortalidad interactuando con los contaminantes.

\section{AGRADECIMIENTOS}

Los autores agradecen a la Consejería de Medio Ambiente y al Registro de Mortalidad de Andalucía la colaboración prestada para la realización de este estudio.

\section{BIBLIOGRAFÍA}

1. Pena González P. La salud y la enfermedad en Huelva en el siglo XIX (1800-1880). Huelva: Diputación de Huelva; 1993.

2. Memoria de Resultados del Programa de Vigilancia y Prevención de la Contaminación Atmosférica correspondiente a 1983 y 1984 . Sevilla: Consejería de Salud y Consumo, Junta de Andalucía; 1985.

3. Medio Ambiente en Andalucía. Informe 89. Sevilla: Consejería de Cultura y Medio Ambiente, Junta de Andalucía; 1990.

4. Escuela Andaluza de Salud Pública. Diagnóstico de salud de la ciudad de Huelva. Granada: Escuela Andaluza de Salud Pública; 1994.

5. Medio Ambiente en Andalucía. Informe 1990. Sevilla: Consejería de Cultura y Medio Ambiente, Junta de Andalucía; 1990.

6. Cepeda Fuentes JM, García Rowe J. Valoración de la Contaminación Atmosférica en el área metropolitana de Huelva en base a bioindicadores liquénicos. Sevilla: Consejería de Medio Ambiente, Junta de Andalucía; 1997.

7. Gravalos Guzmán J, Pereira Vega A, Maldonado Pérez JA, Ramos Sánchez JL, López Tierra G, Pujol de la Llave E. Función pulmonar y síntomas respiratorios en niños de la ciudad de Huelva. An Esp Pediatr 1993 Oct; Suplemento 55:86-91.

8. Lasheras Amat MP. Contaminación atmostërica en la ciudad de Huelva: estudio de correlación ecológica. Granada: Escuela Andaluza de Salud Pública; 1990. 
9. Ballester F, Sáez M, Alonso M, Taracido M, Ordóñez J, Aguinaga I, Daponte A, et al. El proyecto EMECAM: Estudio multicéntrico español sobre la relación entre la contaminación atmosférica y la mortalidad. Antecedentes, participantes, objetivos, material y métodos. Rev Esp Salud Pública 1999; 73:165-175.

10. Pérez-Hoyos S, Sáez M, Barceló MA, Cambra K, Guillén F, Bellido J, Cirera Ll, Ocaña R, et al. Protocolo EMECAM: Análisis del efecto a corto plazo de la contaminación atmosférica so- bre la mortalidad. Rev Esp Salud Pública 1999; $73: 177-185$.

11. World Health Organization. Air quality guidelines for Europe. WHO Regional Publications European Series N. ${ }^{\circ}$ 23. Copenhagen: World Health Organization; 1987.

12. Katsouyanni K, Pantazopoulou A, Touloumi G et al. Evidence for interaction between air pollution and high temperature in the causation of excess mortality. Arch Environ Health 1993; 48: $235-242$. 